\title{
Timing for Elective Cesarean Sections: Have We Got It Right? A 5-year Retrospective Study among Healthy Mothers in Hospital Putrajaya, Malaysia
}

\author{
Salleha Khalid ${ }^{1}$, Hairel ZM Tarmidzi ${ }^{2}$, Norhasniza M Nor ${ }^{3}$, Wan AH Wan Ghazali ${ }^{4}$
}

\begin{abstract}
Introduction: Studies have shown that elective cesarean sections performed before the 39-week period of gestation have increased risks of neonatal morbidity and mortality. Despite this, many elective cases are still performed before this in view of risk associated with emergency cesarean sections. The objective of this study is to determine the neonatal outcome among patients who had elective cesarean section earlier than the 39-week period of gestation.

Materials and methods: Based on the elective cesarean section list from 2013 to 2017, patients with no comorbidity who had elective cesarean section for malpresentation, subfertility, and those who declined the trial of vaginal delivery were identified. The outcome of the delivery particularly looking into neonatal NICU admission and reasons of admission were then explored. Data were analyzed using SPSS version 25.

Results: A total of 435 of $918(47.36 \%)$ healthy patients had elective cesarean sections. There was significant differences in neonatal admission who had cesarean section at 37,38 , and 39 weeks among the healthy $[X 2(4, \mathrm{~N}=438)=10.276, p=0.036]$. There were $26(2.83 \%)$ patients who had an uncomplicated emergency cesarean section prior to an elective date given.

Conclusion: Neonates who were born to healthy mothers had significantly less NICU admissions if they were delivered after the 39-week period of gestation between the patients.

Keywords: Cesarean section, Perinatal outcome, Pregnancy.

Journal of South Asian Federation of Obstetrics and Gynaecology (2020): 10.5005/jp-journals-10006-1773
\end{abstract}

\section{INTRODUCTION}

Infants born before the 39-week gestation point have an increased risk of respiratory complications. ${ }^{1}$ This includes the risk of developing respiratory distress syndrome, newborn transient tachypnea, and an increase in neonatal admissions, to name a few. ${ }^{2}$ As gestational age declines, the morbidity for the infants increases. ${ }^{3}$ When compared to babies born vaginally, babies who born via the cesarean section, especially before the onset of labor, are at increased risk of the adverse respiratory outcome. ${ }^{3}$ This risk continues to be increased in babies who were delivered via the cesarean section, even at full term. ${ }^{1}$

In view of the adverse respiratory outcomes, the Royal College of Obstetricians and Gynecologists (RCOG) has released a green top guideline for the elective cesarean section. ${ }^{4}$ In the guideline, it advocates for the elective cesarean section to be performed at or after the 39-week gestation period. The guideline further adds that should an elective cesarean section need to be done prior to 39 weeks, a course of steroids is advocated to promote lung maturity. ${ }^{4}$

Since the release of the guidelines from the RCOG, the recommendation has changed clinical practices in some centers in Malaysia. Patients in for the elective cesarean section due to obstetric indications such as breech presentation, repeat cesarean section, and those who opted to have cesarean section as their mode of delivery are scheduled to have their elective cesarean section at or after the 39-week gestation point.

As a result, patients will have 1 extraweek compared to the previous practice of having a planned cesarean section between 38 weeks and 39 weeks. Prolonging the date for elective cesarean section up to 39 weeks will risk the patient going into active labor,
${ }^{1}$ Faculty of Medicine and Health Sciences, Kuala Lumpur, Malaysia

${ }^{2-4}$ Department of Obstetrics and Gynecology, Hospital Putrajaya, Putrajaya, Malaysia

Corresponding Author: Salleha Khalid, Faculty of Medicine and Health Sciences, Kuala Lumpur, Malaysia, Phone: +60 376228622, e-mail: sallehakhalid@gmail.com

How to cite this article: Khalid S, Tarmidzi HZM, Nor NM, et al. Timing for Elective Cesarean Sections: Have We Got It Right? A 5-year Retrospective Study among Healthy Mothers in Hospital Putrajaya, Malaysia. J South Asian Feder Obst Gynae 2020;12(2):96-99.

Source of support: Ministry of Health, Malaysia

Conflict of interest: None

which will require an emergency cesarean section to be done immediately as they are already in labor. ${ }^{5}$ This will significantly increase the morbidity rate of both mother and her baby in view of having the cesarean as an emergency rather than as an elective session. ${ }^{6}$ Although the RCOG produced the guideline based on the studies conducted, it may not necessarily reflect a similar picture for the Malaysian population. There are no similar local studies assessing the outcome for the babies delivered before 39 weeks compared to those after 39 weeks up to this date. Whether the increased number of babies admitted to NICU was significantly higher for those delivered at 37 weeks or 38 weeks compared to those delivered after 39 weeks remains to be proven.

The main objective of this study is to determine the neonatal outcome among patients who had an elective cesarean section from 37 completed weeks onward. Specific objectives include determining the prevalence and cause of neonatal admissions 
among patients who had an elective cesarean section from 37 completed weeks onward and to make recommendations on the delivery time for women undergoing elective cesarean section as their mode of delivery.

\section{Materials and Methods}

This is a retrospective study that was conducted from January 1 , 2013, to December 31, 2017, in Hospital Putrajaya. Based on the elective cesarean section list, healthy patients with no comorbidities were identified, as well as those who had an elective cesarean section for malpresentation, previous two or more cesarean sections, and those who requested a cesarean section as their mode of delivery. The outcome of delivery, particularly neonatal NICU admission and the reason for admission, was identified.

The date of gestation was based on the patient's last normal menstrual period. However, if the date was uncertain or due to irregular menses, a revised date of expected delivery was used based on the patient's first trimester scan. The inclusion criteria included women undergoing the elective cesarean section due to the obstetric indications of malpresentation, two or more previous cesarean sections, and declining vaginal delivery. The exclusion criteria included women who had gone through multiple pregnancies, congenital abnormalities, and who had any obstetric or medical condition that warranted that their delivery be excluded from the study, i.e., gestational diabetes and a hypertension complicating pregnancy.

All women who underwent an elective cesarean section due to malpresentation, previous uterine surgery, and those who declined vaginal delivery from January 1, 2013, to December 31, 2017, were included in this study. Based on the Hospital Putrajaya census, we noted that there was an average of 900 deliveries each year with $20 \%$ of women who delivered having had an elective cesarean section. Using OpenEpi version 3, the sample size calculation was as follows:

$$
\text { Sample size } n=[\operatorname{DEFF} * \operatorname{Np}(1-p)] /\left[\left(d^{2} / Z^{2}{ }_{1-\alpha / 2}\right) *(N-1)+p *(1-p)\right] \text { with }
$$

Population size (for finite population correction (FPC) factor) (N): 900

Hypothesized \% frequency of outcome factor in the population (p): $20 \% \pm 5$

Confidence limits as $\%$ of 100 (absolute $\pm \%$ ) $(d): 5 \%$

Design effect (for cluster surveys-DEFF): 1

Confidence level of 95\%, the sample size calculated $=194$ each year

Total sample size $=194 \times 5$ years $(2013-2017)=970$ patients

The outcome was based on the age of gestation at the time of delivery and any neonatal adverse outcomes, i.e., NICU admission, reason for admission, i.e., adverse respiratory outcome such as respiratory distress syndrome and transient tachypnea of the newborn. Other causes of NICU admission include hypoglycemia, newborn sepsis, necrotizing enterocolitis, hypoxic ischemic encephalopathy, needing a ventilator, an Apgar score at 5 minute that is below 3, and an arterial cord pH of below 7. Each of the adverse outcomes above will be assessed against the gestational age at the time of delivery. The maternal outcome was also assessed including the occurrence of an emergency cesarean section being earlier than given date for cesarean section, and if any complications occurred.

The statistical tests were performed using SPSS (version 20, IBM Corporation, Armonk, New York). The categorical data were presented as frequency and percentage. The Pearson's Chi-square or Fisher's exact test was used to analyze the data. Simple logistic regression was used to determine the recommended time for the elective cesarean section. A $p$ value of 0.05 is statistically significant in this study.

\section{Ethical and Humane Considerations}

This research has been registered under the National Medical Research Registry with the registration number of NMRR-17-255638624. The study was granted ethical approval by the Medical Research and Ethics Committee. The data obtained from the patient records are confidential and will be used only for academic purposes and for publication without revealing the patient's identity.

\section{Results}

A total of 918 patients delivered via the elective cesarean section from January 1, 2013, to December 31, 2017, in Hospital Putrajaya. A total of 483 out of 918 (52.61\%) patients were excluded from the study in view of detected maternal or fetal comorbidity. From 483, it was noted that $254 / 483$ (52.59\%) had diabetes during pregnancy, $54 / 483$ (11.18) had hypertensive diseases, and 295/483 (61.08\%) had other illnesses throughout pregnancy. A total of 435/918 (47.39\%) healthy patients were included in this study.

Out of the 435 patients, $68 / 435$ (15.63\%) had a cesarean section between 37 weeks' and 38 weeks' gestation, 332/435 (76.32\%) patients had between 38 weeks and 39 weeks, while 35/435 (8.05\%) patients had a cesarean at or after 39 weeks' gestation. There were $13 / 435$ (2.99\%) neonates admitted to the NICU. Neonatal admission for each gestational week was noted to be $4 / 68$ (5.88\%) and $9 / 332$ (2.71\%) for cesarean section performed at 37-38 weeks and 38-39 weeks, respectively. There were no NICU admissions for those who had a cesarean at 39 weeks and above. These findings were then further analyzed using the Pearson's chi-square test. There were no significant differences in NICU admission among the healthy mothers if the cesarean sections were performed at 37,38 , or at 39 weeks' gestation and above $\left[X^{2}(2, N=435)=3.131\right.$, $p=0.209$ ] (Table 1). Among the 13 neonates who were admitted, 6 had transient tachypnea of the newborn (TTN); another three had congenital pneumonia. The rest was due to various reasons including neonatal jaundice, being small for their gestational age, and admission for further assessment to rule out spinal bifida and congenital adrenal hyperplasia.

Indications for elective cesarean sections included abnormal presentation, previously having had two cesarean sections, and maternal request. The number of cesarean sections performed for each indication against period of gestation is shown in Table 2. While the majority of the elective cesarean sections were performed between 38 weeks' and 39 weeks' gestation, there was a significant relationship between the indications of the elective cesarean section and the timing of the elective cesarean section $\left[X^{2}(4, N=435)=10.276, p=0.036\right]$ where those having had two previous cesarean sections were more likely to have a cesarean at 38-39 weeks compared to the other indications. Among the 35/435 (8\%) patients who had an elective cesarean section at and after 39 weeks, $15 / 35$ (42.86\%) were due to having previous two or more scars, 10/35 (28.57\%) were due to malpresentation, and another $10 / 35(28.57 \%)$ were due to maternal request.

There were $26 / 918$ cases of emergency cesarean sections performed. Among the healthy patients, a total of 11/435 (2.53\%) cases had an emergency cesarean section as their labor started 
Table 1: Neonatal admission based on week of gestation

\begin{tabular}{|c|c|c|c|c|c|c|}
\hline & & & & \multicolumn{2}{|c|}{ Neonatal outcome } & \multirow[b]{2}{*}{ Total } \\
\hline & & & & NICU admission & Discharge to mom & \\
\hline \multirow[t]{6}{*}{ Period of gestation } & \multirow[t]{2}{*}{37 weeks } & \multicolumn{2}{|c|}{ Count } & 4 & 64 & 68 \\
\hline & & \multicolumn{2}{|c|}{$\%$ within period of gestation } & 5.9 & 94.1 & 100.0 \\
\hline & \multirow[t]{2}{*}{38 weeks } & \multicolumn{2}{|c|}{ Count } & 9 & 323 & 332 \\
\hline & & \multicolumn{2}{|c|}{$\%$ within period of gestation } & 2.7 & 97.3 & 100.0 \\
\hline & \multirow[t]{2}{*}{39 weeks } & \multicolumn{2}{|c|}{ Count } & 0 & 35 & 35 \\
\hline & & \multicolumn{2}{|c|}{$\%$ within period of gestation } & 0.0 & 100.0 & 100.0 \\
\hline \multirow[t]{2}{*}{ Total } & & \multicolumn{2}{|c|}{ Count } & 13 & 422 & 435 \\
\hline & & \multicolumn{2}{|c|}{$\%$ within period of gestation } & 3.0 & 97.0 & 100.0 \\
\hline \multicolumn{7}{|c|}{ Chi-square tests } \\
\hline & Value & $d f$ & Asymp. Sig. (two-sided) & Exact Sig. (two-sided) & Exact Sig. (one-sided) & Point probability \\
\hline Pearson Chi-square & $3.131^{\mathrm{a}}$ & 2 & 0.209 & 0.195 & & \\
\hline Likelihood ratio & 3.756 & 2 & 0.153 & 0.206 & & \\
\hline Fisher's exact test & 2.503 & & & 0.256 & & \\
\hline Linear-by-linear association & $3.110^{\mathrm{b}}$ & 1 & 0.078 & 0.087 & 0.069 & 0.049 \\
\hline No. of valid cases & 435 & & & & & \\
\hline
\end{tabular}

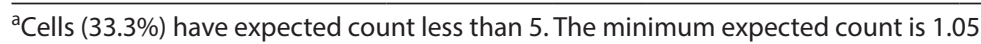

${ }^{\mathrm{b}}$ The standardized statistic is 1.764

Table 2: Indications of elective cesarean section and week of gestation

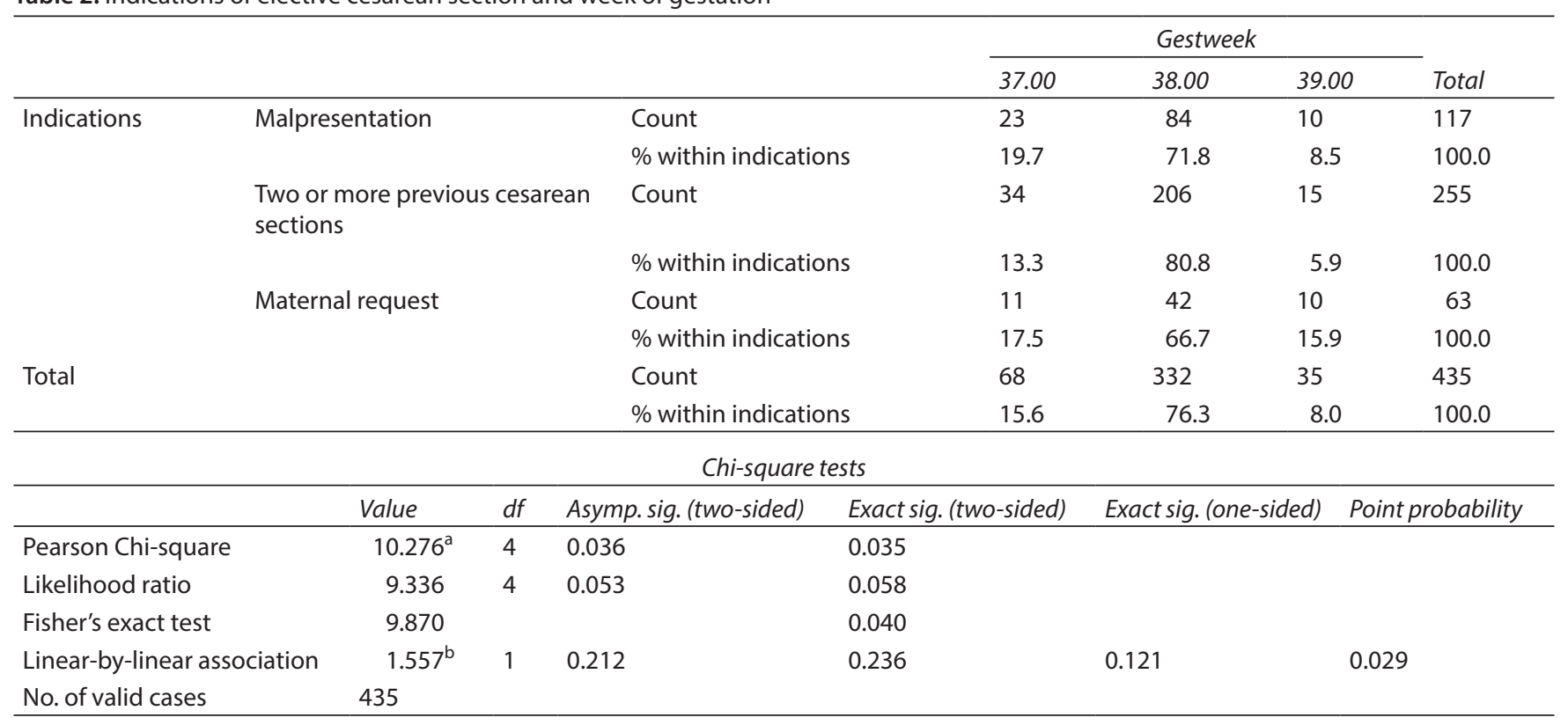

${ }^{\mathrm{a}} 0$ cells $(0.0 \%)$ have expected count less than 5 . The minimum expected count is 5.07

${ }^{\mathrm{b}}$ The standardized statistic is 1.248

earlier than the elective cesarean section dates given earlier. The initial indications for cesarean sections were $4 / 11$ (36.4\%) for malpresentation, 4/11 (36.4\%) for maternal request, and 3/11 (37.2\%) for having had two or more previous cesarean sections. There were no complications documented among mothers who had an emergency cesarean section. There was 1 NICU admission out of the 11 cases of emergency cesarean section. The indication for NICU admission was presumed sepsis. The baby was discharged after completing a 24-hour course of antibiotics.

\section{Discussion}

The number of cesarean sections has increased over the period of time. ${ }^{5}$ The benefit of delivering babies after 39 weeks' gestation has been shown in many studies. Having an elective cesarean section before 39 weeks has been shown to be associated with an increase in respiratory morbidity among neonates. So much so, that it has been recommended that should an elective cesarean section need to be performed before 39 weeks, a course of 
steroids needs to be completed prior to delivery ${ }^{4}$ in order to ensure lung maturity. However, this has not changed the clinical practice in Malaysia as many still perform an elective cesarean section between 38 weeks and 39 weeks. Many clinicians were concerned and prefer to do it earlier in view of the risk of having an emergency procedure if the labor started earlier than the elective cesarean dates. Our results of a retrospective review in Hospital Putrajaya, however, did not support the findings. There were no differences in the outcome of the neonates when undergoing an elective cesarean section at 37-38 weeks, 38-39 weeks, or 39 weeks and onward. There were only 13 NICU admissions among the 435 healthy patients, with no admission among those who had a cesarean at 39 weeks and above. These findings are not significant. The small number of NICU admissions may not be surprising as this could be due to the fact that this study only involved healthy mothers. Having no comorbidity such as diabetes in pregnancy, hypertensive disease, or anemia among the mothers and a well-planned cesarean section means that one can only expect a good outcome. Nevertheless, among the 13 NICU admissions, 9 of the admissions were due to respiratory illness. This reflects that respiratory morbidity is still the main reason for neonatal admission to the NICU among those who had an elective delivery prior to 39 weeks.

The majority of the elective cesarean sections were performed between 38 weeks and 39 weeks. Women who had two or more scars as the initial indication for cesarean were more likely to have their elective date within 38-39 weeks compared to the other indications. This may be due to the fact that the risk of scar dehiscence increases with each cesarean section. The risk of scar dehiscence was reported to range from 0.2 to $4.6 \%$ among women who have one cesarean scar (3). This risk is expected to be much higher among women who have more than one scar. In view of this, many clinicians prefer to put an earlier elective date before 39 weeks among patients who have had two or more cesarean sections. Interestingly, despite the risk associated with emergency cesarean sections, there were 35 women who had a cesarean at 39 weeks or above. Fifteen of the cases had an elective cesarean due to having two previous scars. Although those who had two previous scars were more likely to have a cesarean date between 38 weeks and 39 weeks, there were still cases who had a cesarean at or after 39 weeks' gestation. This can be due to the fact that there were not enough slots available for a cesarean to be performed before 39 weeks. On the other hand, if elective cases were only conducted at 39 weeks and above, there may not be enough slots for an elective cesarean to fit in within a short duration of time compared to the 2 weeks' duration if the elective cesarean was performed at 38 weeks and above. Due to this limited amount of time, some patients would inevitably have their cesarean before 39 weeks.
Emergency cesarean sections were noted to be at $2.53 \%$, which is far less than reported elsewhere, reaching $10 \% .^{7}$ The risk associated with emergency cesareans should not be disregarded. ${ }^{6}$ The benefit of reducing neonatal morbidity from $11 \%$ at 38 weeks to $8 \%$ at 39 weeks 1 needs to be weighed against the maternal and neonatal morbidity associated with emergency cesarean sections as a result of delaying delivery up to 39 weeks and above.

This is a retrospective study and, by having a retrospective nature, some data may have been lost or was wrongly entered in the system. This is a single-center study. A multicenter study needs to be performed in the future to ensure that the data and results gained will be truly representative of the whole population in Malaysia.

\section{Conclusion}

In summary, from the data above, there was no significant difference in neonatal outcome if the elective cesarean section was performed at 37-38 weeks, 38-39 weeks, or 39 weeks and beyond. Nevertheless, a bigger prospective study involving multiple centers needs to be performed to confirm the findings.

\section{Clinical Significance}

Having elective cesarean section before 39 weeks' gestation may still be acceptable as there was no significance difference in neonatal admission between those delivered before and after 39 weeks' gestation.

\section{References}

1. Tita $A T$, Landon MB, Spong CY, et al. Timing of elective repeat cesarean delivery at term and neonatal outcomes. New Eng J Med 2009;360(2):111-120. DOI: 10.1056/NEJMoa0803267.

2. Morrison JJ, Rennie JM, Milton PJ. Neonatal respiratory morbidity and mode of delivery at term: influence of timing of elective caesarean section. Br J Obstet Gynaecol 1995;102(2):101-106. DOI: 10.1111/j.14710528.1995.tb09060.x.

3. Zanardo V, Simbi AK, Franzoi M, et al. Neonatal respiratory morbidity risk and mode of delivery at term: influence of timing of elective caesarean delivery. Acta paediatrica 2004;93(5):643-647. DOI: 10.1111/j.1651-2227.2004.tb02990.x.

4. No GT. Placenta praevia, placenta praevia accreta and vasa praevia: diagnosis and management. London: RCOG; 2011. pp. 1-26.

5. Baron J, Weintraub AY, Eshkoli T, et al. The consequences of previous uterine scar dehiscence and cesarean delivery on subsequent births. Int J Gynecol Obstet 2014;126(2):120-122. DOI: 10.1016/ j.ijgo.2014.02.022.

6. Pallasmaa N, Ekblad U, Gissler M. Severe maternal morbidity and the mode of delivery. Acta Obstet Gynecol Scand 2008;87(6):662-668. DOI: 10.1080/00016340802108763.

7. Thomas J, Paranjothy S. The national sentinel caesarean section audit report. RCOG press; 2001. 